\title{
Reactive power management of a AC/DC microgrid system using a smart PV inverter
}

\author{
Fida Hasan Md Rafi, Student member, IEEE, M. J. Hossain, Senior Member, IEEE, D. Leskarac, J. Lu, Senior Member, \\ IEEE \\ Queensland Micro and Nano Technology Center, School of Engineering, Griffith University, Gold coast, Australia \\ Fida.rafi@griffithuni.edu.au, j.hossain@griffith.edu.au, d.leskarac@griffith.edu.au, j.lu@griffith.edu.au
}

\begin{abstract}
This paper analyses the effect of a smart voltage source inverter (VSI) embedded in a photovoltaic (PV) unit on the voltage stability/reactive power balancing of a microgrid. The conventional unity power factor PV-VSI is modified to operate for both active and reactive power management operations. The PV integrated AC/DC microgrid system is designed with a central smart VSI in PSCAD/EMTDC software environment and implemented in the Energex $11 \mathrm{kV}$ distribution network, Australia. The results show superior dynamic performance and robust transient recovery from the smart VSI integrated microgrid for real life radiation variations, random loads variations, faults and intentional external disturbances.
\end{abstract}

Index Terms-- PV microgrid, AC/DC microgrid, smart VSI, VAR compensation, PV-STATCOM.

\section{INTRODUCTION}

Renewable energy sources are gaining much popularity throughout the word in the electrical power generation sectors. Among the popular sustainable energy sources, PV system is the most popular one, as small scale PV can easily be installed in consumer level. Additionally, special government incentives and feed in tariff opportunities are available with the PV installations in different developed countries. However, the proliferation of PV system integrations with the distribution network has considerable effects on the power transfer capability of distribution lines. Generally, difference reactive power management devices like series compensation devices, flexible AC transmission system devices (FACTS), static capacitors, tap changing transformers etc. are used to improve the power transfer limits of the distribution networks [1]. The number of PV system integrations is higher in the low voltage (LV) distribution network than in the medium or high voltage networks. Therefore, among the conventional reactive power compensation devices, a few are practically applicable in LV distribution network like the distribution static synchronous compensator (d-STATCOM), switched capacitor bank and tap-changing transformer. The d-STATCOM is the most viable option considering modern power system dynamic characteristics, nevertheless, the economic issue is still the main impediment on its vast implementation in the distribution network.

The basic operation of the d-STATCOM is to supply/draw reactive current from the utility grid lines at the point of common coupling (PCC) to improve voltage regulation and enhance damping operation via proper reactive power (VAR) management. As, the core component for the d-STATCOM and the PV system is the VSI, many researchers are working to utilize the VSI from PV inverter to have decentralized reactive power capability similarly to the d-STATCOM along with conventional PV active power operation. However, the IEEE 929 and 1547 standards require the PV units to operate at unity power factor in the point of common coupling (PCC) [2] [3]. But, considering the significant PV integrations and total power generations worldwide, around 139 GW [4], top PV installation country, Germany, and recent IEEE standard (1547.8) has already introduced the reactive power control operations for the grid-tied PV system [5][6].

The concept of utilizing the solar PV inverters as STATCOM is presented by the authors in [7], where they evaluated the performance with fixed number of loads variations. Different reactive power operations for voltage sag/swell due to high PV penetrations in the low voltage (LV) distribution network have been analyzed in [8] [9] [10]. The issues with cloud passing and divergent radiation effects are mainly analyzed in those papers. Authors in [11] presented improved distribution level loading capacity under cloud passing condition with the reactive power operation from a single phase PV VSI. Without considering the PV nonlinearities and load variations, the voltage imbalanced at the PCC point has been improved following VDE-AR-N 4105 and German Association of Energy and Water Industries (BDEW) code in [12]. A decentralized robust controller is presented to reduce distribution network oscillation due to close PV unit's controllers' interaction in the AC network in [13]. Different power management operations in the AC/DC microgrid system are available in [14] [15], however, there are no clear indication about the power interaction effects with the real solar radiation data or how the system will interact without additional islanding controllers. Generally the PV inverter capacity is chosen 

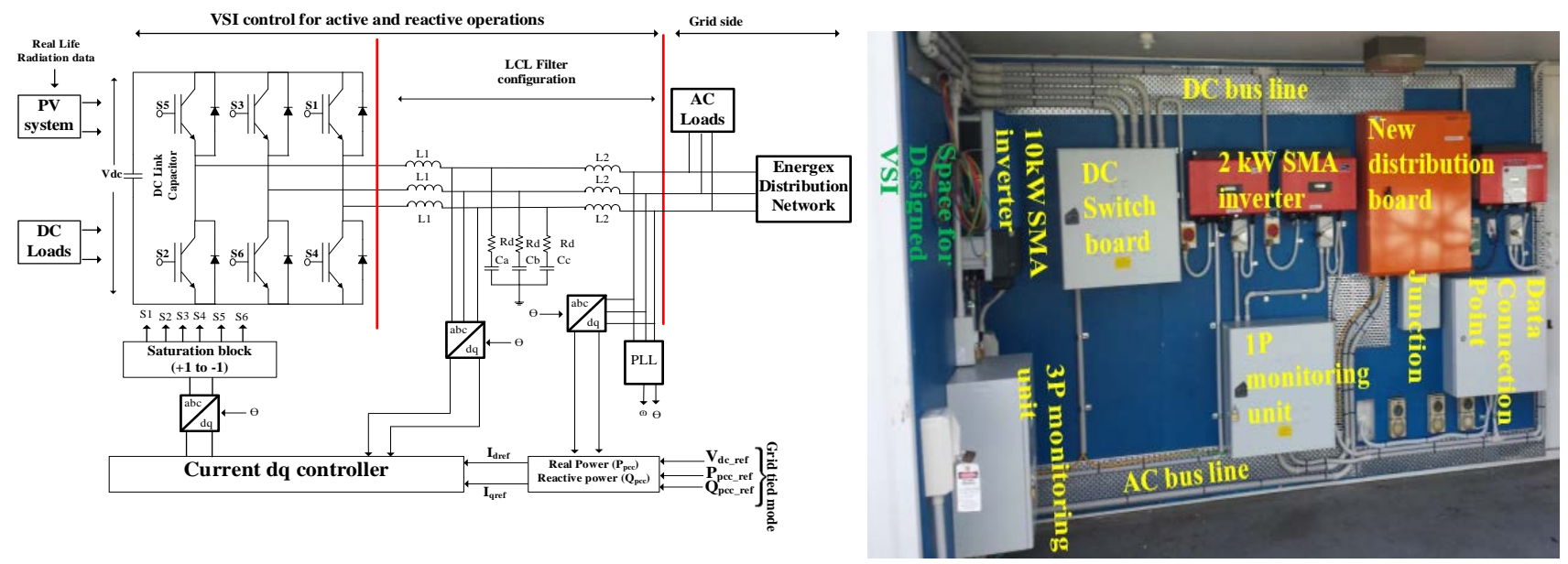

Figure 1. Designed PV microgrid system( left) and Control set up of the real PV microgrid in Griffith University , Australia (right).

to compensate maximum peak demand in households. During sunny days or low loads condition, the remaining capacity after load compensation can easily be utilized by the PV system VSI for reactive power managements at the PCC. This operation also requires dynamic reactive power support during voltage excursions. Some PV inverter manufacturers have already "de-rated" their inverters and now provide both $\mathrm{kW}$ and KVA ratings [16].

Therefore, in this paper, the dynamic stability and robust stabilization recovery has been compared with the conventional PV inverter and designed PV VSI with additional VAR compensation operation in the AC/DC microgrid connected with the real distribution network of Energex, Queensland, Australia. The analysis is carried out in several cases, for example, real life radiation variations and different static loads changing, different faults effects, and intentional islanding effects without separate islanding controller. The comparison results prove the improved controller performance as well as robust stabilization from the modified VSI of the PV system.

\section{SYSTEM DESCRIPTION}

The designed microgrid system consists of DC and AC buses with a central smart VSI for the coordination of real and reactive power at the PCC. The system is designed resembling the real ongoing smart microgrid project of Griffith University, Australia. The project consists of a 10 kW PV system, $30 \mathrm{kVA}$ four quadrant operable smart VSI and $60 \mathrm{kWh}$ Li-ion battery storage unit. The main purpose of the real project is to compare the results from the conventional SMA PV inverters with the modeled smart VSI. The four quadrant operations from the designed VSI utilizing PV generated excess power in the AC/DC microgrid is presented in [15]. The designed PV microgrid model and the control panel from the real project are shown in Fig. 1. The designed model parameters are shown in the appendix. The single stage PV system radiation data is taken from $25^{\text {th }}$ September (5 am to 3 $\mathrm{pm}), 2014$ from the real project output. A constant $2 \mathrm{~kW}$ DC load is designed with pure resistive unit to monitor the impact on the DC side as well. As, the analysis of the reactive power operation from the PV VSI is the main aim in this paper, conventional $d q 0$ controller for the outer DC voltage regulator and inner current controllers are designed and applied in this AC/DC microgrid model [18] [19]. Additional load VAR compensation loop is designed to generate the reactive current reference for the smart VSI. The main switching operations are,

$$
\begin{aligned}
& S_{d}=\frac{2}{V_{d c}}\left(L \frac{d i_{d}}{d t}+R i_{d}-L \omega i_{q}+V_{s d}\right) \\
& S_{q}=\frac{2}{V_{d c}}\left(L \frac{d i_{q}}{d t}+R i_{d}+L \omega i_{d}+V_{s q}\right)
\end{aligned}
$$

Where, the symbols carry their standard meanings.

The four quadrant control operations with the conventional VSI with small energy storage like capacitor, requires higher DC voltage than calculated value (around $400 \mathrm{~V}$ ) to ensure proper decoupled control over active and reactive parameters [18]. Elevare, a local energy company and joint member of the real microgrid project, has applied for the patent on the four quadrant STATCOM keeping the DC bus voltage from $600-800 \mathrm{~V}$ to reduce higher DC side current and power losses. Therefore, the DC bus voltage is regulated at constant $800 \mathrm{~V}$ in the designed model. The controllers' proportional-integral (PI) gain values are initially tuned via symmetrical optimum method and then fined tuned to get stabilized performance from the system. The LCL filter with passive damping resistor is utilized to get superior damping capability in the microgrid [20]. Different static load models like constant impedance (Z) and constant power (P) are designed and connected at the PCC. The Newmarket zone substation $11 \mathrm{kV}$ feeder of Energex consists of 2,373 customers and the installed load capacity is $8,954 \mathrm{kVA}$. The detailed connected portion from $33 \mathrm{kV}$ upstream network to $11 \mathrm{kV}$ and $420 \mathrm{~V}$ is shown in the appendix.

\section{CASE STUDIES}

The case studies are carried out to compare the performances between conventional and modified PV VSI operation under real life radiation and random static load 
variations, different faults effects and application of severe external disturbance via intentional islanding operation without separate islanding controller. It is estimated that under standard testing condition (STC), the default PSCAD master library PV module generates around 650 $\mathrm{W}$. The peak voltage and current of a single PV module consisting 108 cells in series and 4 cells in parallel are around $81 \mathrm{~V}$ and $8.125 \mathrm{~A}$. Total 10 parallel and 2 series modules are considered to generate maximum $13 \mathrm{~kW}$ at STC. The DC voltage reference is fixed at $800 \mathrm{~V}$. A $2 \mathrm{~kW}$ DC and divergent static three phase AC loads are connected with the microgrid. The applied loads values are shown in the Table 1 . The case studies show the dynamic stability improvement, wide range of controllability options and robust fault recovery performance from the designed smart VSI. Proper decentralized control over active and reactive power in the microgrid ensures stable supply and demand relationship and exhibits well damped characteristics utilizing the four quadrant operations from the designed VSI even in severe external disturbances.

\section{A. Radiation variations and load changing effects}

In real life environmental conditions, the solar radiation follows nonlinear characteristics. The divergent radiation variations causes voltage sag/swell in the PCC point, resulting higher harmonics and lower power quality from the PV system. Excessive reverse power flow from the PV system during sunny day and low load conditions, can even trip the customer from the utility grid due to voltage rise. Therefore, the system needs to be well damped and stabilized to withstand divergent radiations cases. Similar effects can be explained for random loads variations. But, the load peak demand can be predicted monitoring overall load usage from the past. Despite the prediction case, randomly divergent loads connection and disconnection can cause transient phenomenon at the PCC. It is the responsibility of the microgrid/ customers to maintain proper demand and supply relationship even in unpredictable load variations.

Therefore, the designed microgrid radiation data is taken from the real microgrid project on $25^{\text {th }}$ September, 2014 and six pairs of loads combinations are used with the system. The motor characteristics is represented in static nature by the constant power $(\mathrm{P})$ load and some other most commonly used loads like incandescent light, water heater etc. are represented by the static impedance $(\mathrm{Z})$ load. The static load characteristics can be represented as [19]:

$$
\begin{aligned}
\mathrm{P}_{\text {load }} & =\mathrm{P}_{0}+\left(\mathrm{V} / \mathrm{V}_{0}\right)^{\mathrm{a}} \\
\mathrm{Q}_{\text {load }} & =\mathrm{Q}_{0}+\left(\mathrm{V} / \mathrm{V}_{0}\right)^{\mathrm{b}}
\end{aligned}
$$

where, the constants ' $a$ ' and ' $b$ ' defines the load characteristics. For constant power loads, ' $a$ ' and 'b' equals 0 and for constant impedance loads, ' $a$ ' and ' $b$ ' equals 2 .

\section{TABLE I. VARIABLE LOADS}

\begin{tabular}{|c|c|c|c|c|}
\hline Load (k) & Type & Connect & Disconnect & Duration (s) \\
\hline $6+\mathrm{j} 3$ & Z1 & 6.00 & 6.30 & 0.30 \\
\hline $8+\mathrm{j} 5$ & $\mathrm{P} 1$ & 6.40 & 7.10 & 0.30 \\
\hline
\end{tabular}

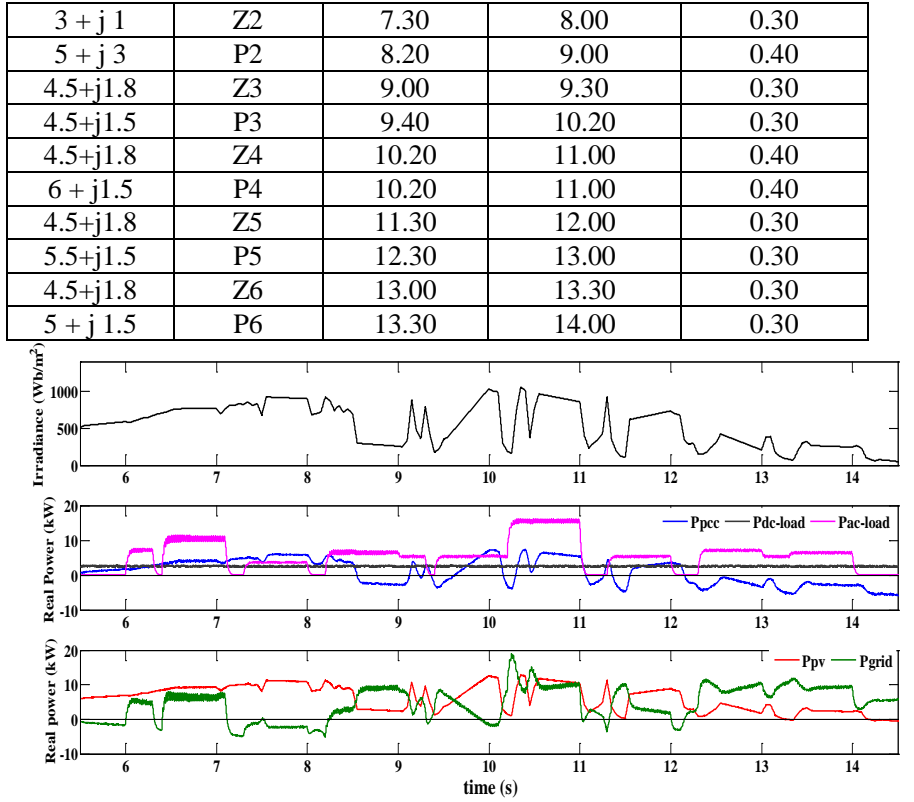

Figure 2. Real power profiles within microgrid

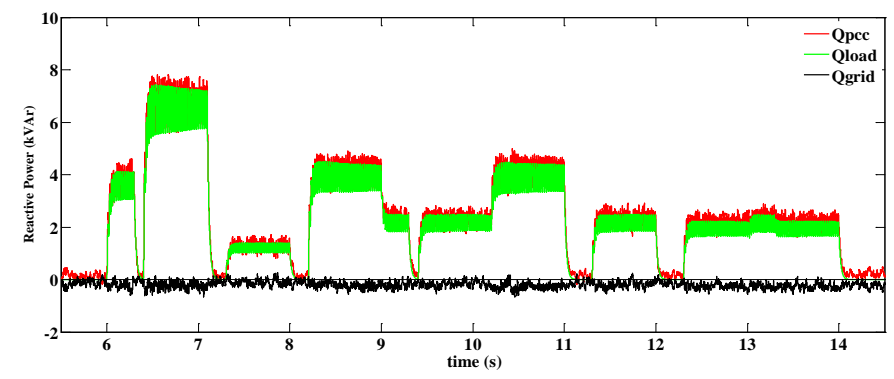

Figure 3. Reactive power profiles within microgrid

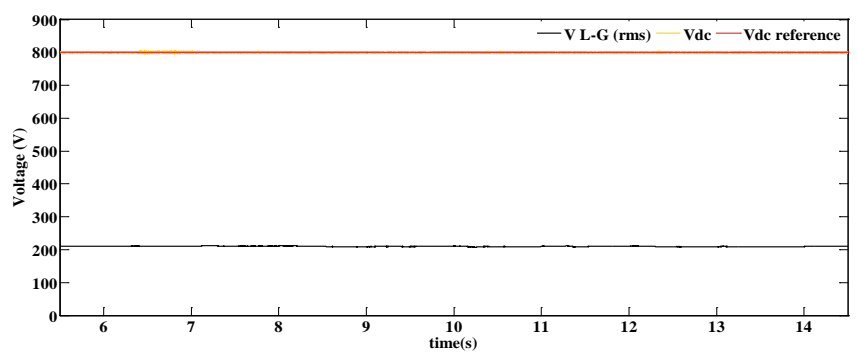

Figure 4. DC bus and V(L-N) rms volatges

The AC loads are varied randomly as shown in Table 1. The radiation data is taken from 5 am to $3 \mathrm{pm}$ and in this case study, the individual hour data is modified for simulation plot in seconds. From Fig. 1 and 2, it is evident that, despite the divergent radiation characteristics and random AC loads variation on the microgrid, designed VSI co-ordinates proper load demand and supply relationship without introducing any instability issue in the overall system. At, $10.20-11.00 \mathrm{~s}$, mixed load is applied with fluctuating radiation variations. Despite the most extreme loads and radiation conditions, the system does not cause any sever voltage sag/swell. The static power load tends to sag the rms voltage but the designed VSI VAR 
compensation operation prevents that to exceed standard limit ( $\pm 10 \%)$ as shown in Fig. 4. The oscillation in the reactive power is due to the inductive nature of the loads and the designed VSI regulates it within marginal range to keep the system stable and well damped.

\section{B. Different faults effects}

This case study demonstrates the fault recovery time improvement utilizing VAR compensation and passive damping from the designed VSI in response to the symmetrical (three phase to ground (3P-G)) and asymmetrical (single phase to ground (1P-G)) faults. A 2 kW DC load and a static constant impedance AC load (6 $\mathrm{kW}+\mathrm{j} 3 \mathrm{kVAR}$ ) are kept connected during this fault analysis study. The system is considered to be at STC.

The occurrence of most severe three phase faults is a common phenomena in distribution level, and the connected systems, either loads or generation units are manufactured to continue their normal operation instantly after the fault clearance.Therefore, a three phase to ground fault is applied after the PCC point from $0.55 \mathrm{~s}-0.75 \mathrm{~s}$ and the after effects of the fault are analyzed with and without the reactive power operation from the VSI .
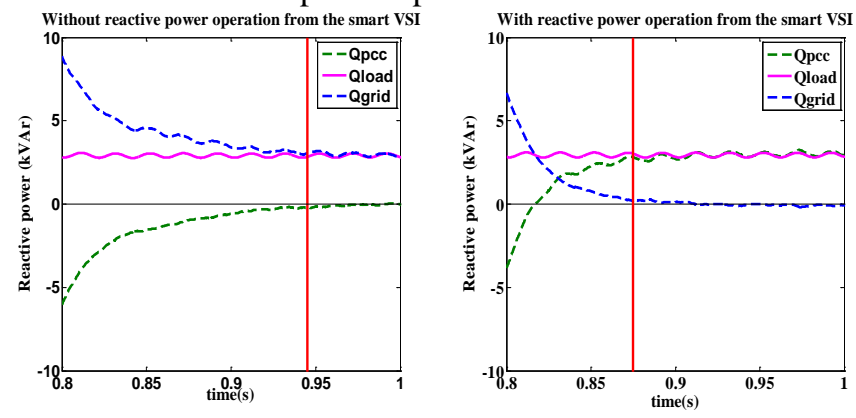

Figure 5. 3P fault recovery response comparison.

The fault is cleared at $0.75 \mathrm{~s}$ and without the reactive power operation, controller reactive part starts to control the system reactive power demand around $0.95 \mathrm{~s}$. However, with the reactive power operation from the designed VSI, the controllers starts controlling around 0.86 $\mathrm{s}$, about 10 milliseconds earlier than without reactive power operation. The significant fault recovery time improvement is due to the decentralized control over both the power profiles. Considering real life dynamic loads and increasing PV penetration numbers, the faster fault recovery response can play vital part for stability enhancement in the connected distribution network.

Similar improvements can be observed for single phase fault case. A single phase fault (B phase to ground) is applied from $1.40 \mathrm{~s}-1.60 \mathrm{~s}$. The cotroller robust fault recovery effetcs are shwon in Fig. 6. The reactive power operations from the VSI also improves the power quality in the microgrid as shwon in Fig. 7 and it is evident that, the designed VSI supplies less harmonics output current from the PV microgrid system. This is due to the improved passive damping capability from the designed VSI which helps to reduce power oscillations in the system.
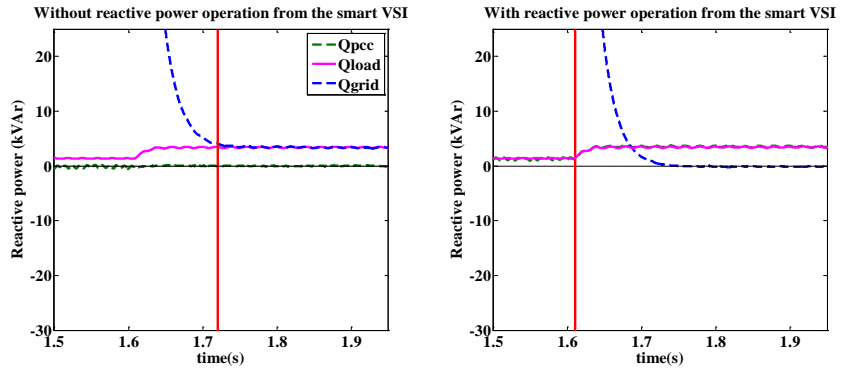

Figure 6. 1P fault recovery response comparison.
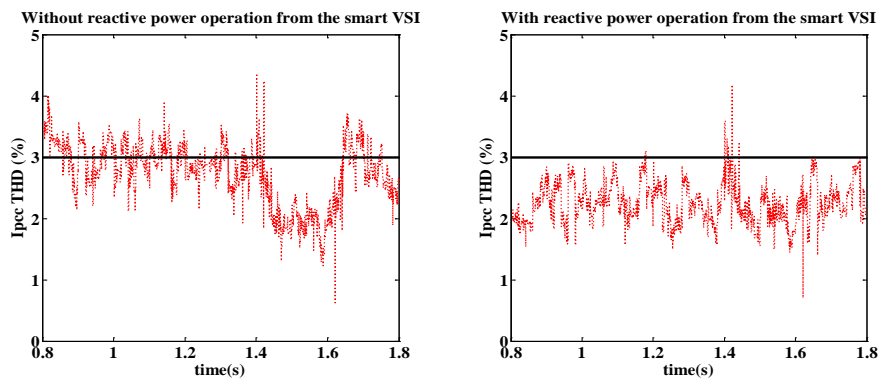

Figure 7. Ipcc THD comparison for $3 \mathrm{P}$ and $1 \mathrm{P}$ faults.

\section{Intentional Islanding scenario}

Generally, renewable sources microgrid systems are designed to operate both in grid-tied and islanded modes. During grid-tied mode the system frequency and voltage magnitude are controlled by the utility grid, however, during islanded condition these two parameters has to be controlled separately by the DG units. Therefore, if some systems are designed not to operate independently during islanding scenario, having reactive operation from the smart VSI can improve the post disturbance recovery response time as well as can damp the system oscillation more quickly after the reconnection with the utility grid. For this case analysis, a $6 \mathrm{~kW}+\mathrm{j} 3 \mathrm{kVAR}$ constant power load is kept connected with the microgrid and the grid side breaker is intentionally disconnected from $0.50 \mathrm{~s}-0.70 \mathrm{~s}$. During and after the islanding case, the conventional PV microgrid requries more VAR support and casues more oscillation in the output power. Whereas, the designed VSI shows less oscillation with robust stablization recovery as shwon in Fig. 8. The additional reactive power operation from the conventional VSI also improves the system frequency recovery time as shown in Fig. 9.
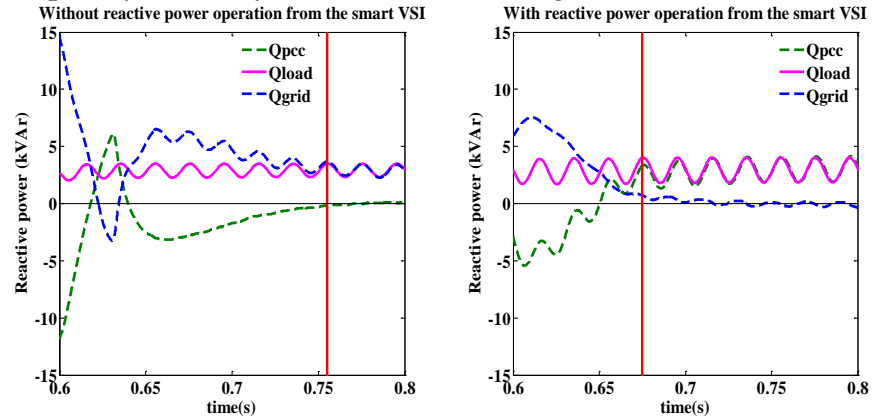

Figure 8. Disturbance rejection response comparison. 


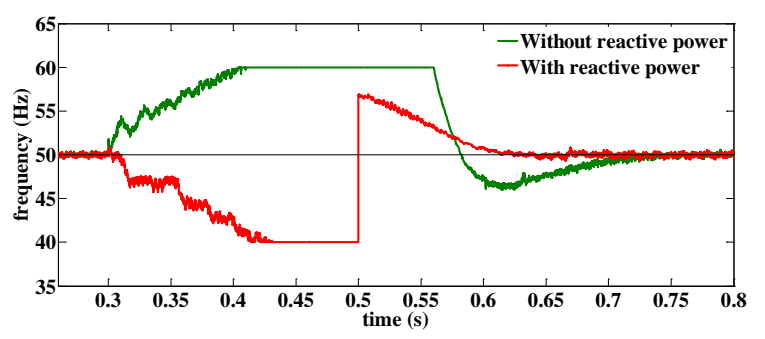

Figure 9. System frequency response comparison from the external disturbance.

\section{CONCLUSION}

The designed PV system VSI certainly shows superior performance improvement in the AC/DC microgrid operations. The controller response speed and system damping capability improves in 10-15 milliseconds range with the additional reactive power and improved passive damping features from the VSI. These features help to achieve proper decentralized control over real and reactive powers in the microgrid, and ensure robust stabilization recovery from externals disturbances. For future studies, multiple PV units with load sharing controls with dynamic loads will be analyzed.

\section{ACKNOWLEDGEMENT}

Author is thankful to ENERGEX, the electric power distribution company owned by the federal government of Queensland, Australia, for providing the distribution network model for this research study.

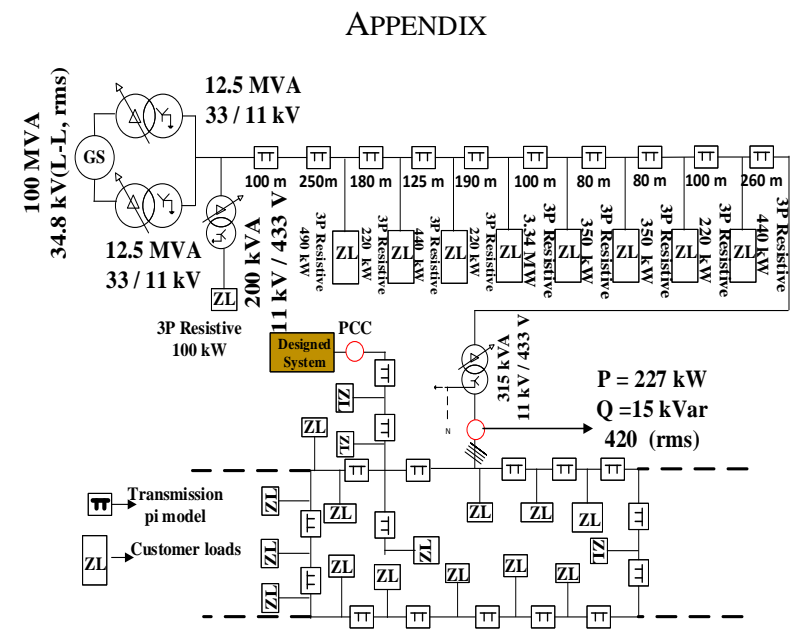

Figure 10. From upstream to Energex LV distribution network.

System description: PV module : 10 series and 2 paraller modules; DC link capacitor $=3000 \mu \mathrm{F}$, switching frequency $=3 \mathrm{kHz}$, Inverter capacity $20 \mathrm{kVA}$; DC voltgae regulator : $\mathrm{Kp}=4, \mathrm{Ti}=0.8$; Current controller : $\mathrm{Kp}=8, \mathrm{Ti}=0.003$; LCL filter : inverter side $\mathrm{L}=4 \mathrm{mH}, \mathrm{C}=10 \mu \mathrm{F}$, grid side $\mathrm{L}=1 \mathrm{mH}$, damping resistor $3 \mathrm{~m} \Omega$; Distibution grid: $240(\mathrm{~L}-\mathrm{N})$ $/ 420$ (L-L) rms , $50 \mathrm{~Hz}$.

\section{REFERENCES}

[1] N. G. Hingorani, and L. Gyugyi, "Understanding FACTS," IEEE press, 1996.

[2] IEEE Recommended Practice for Utility Interface of Photovoltaic (PV) Systems, IEEE Std. 929-2000, 2000.

[3] IEEE Standard for Interconnecting Distributed Resources with Electric Power Systems, IEEE Std. 1547-2003, 2003, pp. 0_1-16.

[4] "Renewables 2014: Global Status Report," REN21, 2014.

[5] Technical Guideline on Power Generation Systems on Medium Voltage Networks-Guideline for Connection and Parallel Operation of Generating Plants to Medium Voltage Network, Bundesverband der Energie- und Wasserwirtschaft e.V., Berlin, Germany, 2008.

[6] http://grouper.ieee.org/groups/scc21/1547.8/1547.8_index.html, IEEE P1547.8 Recommended Practice for Establishing Methods and Procedures that Provide Supplemental Support for Implementation Strategies for Expanded Use of IEEE Standard 1547.

[7] R. K. Varma, S. R. Rangarajan, I. Axente, and V. Sharma, "Novel application of a PV solar plant as STATCOM during night and day in a distribution utility network," IEEE Power Systems Conference and Exposition (PSCE), pp. 1-8, USA, 2011.

[8] K. Turitsyn, P. Sulc, S.Backhaus, M. Chertkov, "Options for Control of Reactive Power by Distributed Photovoltaic Generators," Proceeding of IEEE, vol. 99 , issue 6, pp. 1063-1073, 2011.

[9] E. Demirok, P. C. Gonzalez, K. H. B. Frederiksen, D. Sera, P. Rodriguez, and R. Teodorescu, "Local Reactive Power Control Methods for Overvoltage Prevention of Distributed Solar Inverters in Low-Voltage Grids," IEEE Journal of Photovoltaics, vol. 1, no. 2, pp. 174-182, 2011.

[10] A. Samadi, M. Ghandhari, and L. Soder, "Reactive power dynamics assessment of a PV system in a distribution grid," Energy Procedia, vol. 20, pp. 98-107, 2012.

[11] R. Yan and T. K. Saha, "Investigation of Voltage Stability for Residential Customers Due to High Photovoltaic Penetrations," IEEE Trans. on Power System, vol. 27, no. 2, pp. 651-662, 2012.

[12] Y. Bae, T. K. bu, and R. Y. Kim, "Implemental Control Strategy for Grid Stabilization of Grid-Connected PV System Based on German Grid Code in Symmetrical Low-to-Medium Voltage Network," IEEE Trans. on Energy Conversion, vol. 28, no. 3 , pp. 619-631, 2013.

[13] M. J. Hossain, M. A. Mahmud, H. R. Pota, and N. Mithulananthan, "Design of Non-Interacting Controllers for PV Systems in Distribution Networks," IEEE Trans. on Power Systems, issue 99, pp. 1-12, April, 2014.

[14] X. Liu, P. Wang, and P. C. Loh, "A hybrid AC/DC microgrid and its coordination control,” IEEE Trans. on Smart Grid, vol. 2, no. 2, pp. 278-286, June, 2011.

[15] N. Eghtedarpour and E. Farjah, "Power Control and Management in a Hybrid AC/DC Microgrid”, IEEE Trans. on Smart Grid, vol. 5 (3), pp. 1494-1505, April, 2014.

[16] A. Ellis, R. Nelson, E. V. Engeln, R. Walling , J. M. Dowell , etc. all, "Reactive Power Interconnection Requirements for PV and Wind Plants-Recommendations to NERC “, Sandia National laboratory (SAND2012-1098) 2012.

[17] F. H. M. Rafi, J. Hossain, and J. Lu, "Design of a Single Stage Transformerless VSI in a Smart Microgrid for PV-STATCOM/ESS operations," Australasian University Power Engineering Conference, Perth, Australia, 2014.

[18] C. Schauder and H. Mehta, "Vector analysis and control of advanced static VAr compensators", IEE Proceedings-C: Generation, Transmission and Distribution, pp. 299-306, 1993.

[19] A. Yazdani, and R. Iravani, "Voltage-sourced converters: Modeling, control, and application,” Hoboken, NJ: Wiley, 2010.

[20] A. Reznik, M. Godoy, A. A. Durra, and S. M. Muyeen, "LCL filter design and performance analysis for grid interconnected systems," IEEE Trans. on Industry Applications, vol. 50, no. 2, pp. 12251232, March/April, 2014.

[21] P. Kundur, "Power System Stability and Control", McGraw Hill Inc., 1994. 